\title{
FITOSSOCIOLOGIA E ESTRUTURA DIAMÉTRICA DE UM FRAGMENTO DE CERRADO SENSU STRICTO, GURUPI - TO
}

Phytosociology and Diameter Structure of a Cerrado Sensu Stricto, Gurupi - TO

Fitosociológico y Estructura Diamétrica um Fragmento Cerrado Sensu Stricto, Gurupi-TO

\author{
Gabriel Oliveira Silva $^{* 1}$, Priscila Bezerra Souza ${ }^{1}$ \\ ${ }^{1}$ Curso de Engenharia Florestal, Campus de Gurupi, Tocantins, Brasil. \\ *Correspondência: Rua Badejós, Lote 7, Chácaras 69/72-Zona Rural| Caixa Postal 66- 77402-970 . e-mail \\ gabrieloliveira_@hotmail.com
}

Artigo recebido em 30/10/2015. Aprovado em 09/12/2015. Publicado em 24/02/2017.

\section{RESUMO}

Objetivou-se realizar um levantamento fitossociológico e determinar a estrutura diamétrica de uma área de cerrado sensu stricto. $\mathrm{O}$ estudo foi conduzido numa fisionomia de cerrado sensu stricto, Reserva Legal da Fazenda Experimental da UFT, campus de Gurupi, sob as coordenadas geográficas $11^{\circ} 77^{\prime} 30.5^{\prime \prime} \mathrm{S}$ e $49^{\circ} 05^{\prime} 62.9^{\prime \prime} \mathrm{W}$. Foram instaladas sistematicamente cinco parcelas de 20x50m $\left(1000 \mathrm{~m}^{2}\right)$ cada, sendo distanciadas $10 \mathrm{~m}$ entre si, perfazendo um total de $5000 \mathrm{~m}^{2}$ de área amostral. Nessas parcelas foram amostrados todos os indivíduos arbustivo-arbóreos com circunferência altura do peito $(\mathrm{CAP}) \geq$ a $10 \mathrm{~cm}$. Foram amostrados 2128 indivíduos, dos quais 2062 vivos e 66 mortos em pé, distribuídos em 110 espécies 86 gêneros e 46 famílias. A altura média da vegetação estimada foi de $7,23 \mathrm{~m}$ o diâmetro médio encontrado foi de $6,87 \mathrm{~cm}$ além de ter apresentado uma área basal total de $10,88 \mathrm{~m}^{2}$. O índice de diversidade de Shannon (H') foi de 3,65 e equabilidade de Pielou (J') 0,775. Foi observada a presença do padrão de distribuição diamétrica presentes nesta comunidade vegetal, em J invertido.

Palavras-chave: Estrutura vegetacional, J invertido, Cerrado.

\section{ABSTRACT}

This aimed at carrying a phytosociological survey and determine the diametric structure of a cerrado 'stricto sensu'. This study was conducted in Gurupi - TO, in the UFT Experimental Farm at Gurupi Campus, being the studied area inside a legal reserve located under the geographic coordinates 11"77'30.5 S e 49"05'62.9 W. Five plots of $20 \times 50 \mathrm{~m}, 10 \mathrm{~m}$ away from each other, making $5000 \mathrm{~m}^{2}$ or 0,5 ha of sampled area were installed. Inside this area all shrub-arboreal individuals with $C A P \geq 10 \mathrm{~cm}$ were sampled. A total of 2128 individuals were sampled, with 2062 living and 66 standing dead, over 110 species, 42 families and 86 genera. The average height was of 7 , $23 \mathrm{~m}$, mean diameter of $6,87 \mathrm{~cm}$ and basal area of $10,88 \mathrm{~m}^{2}$. The Shannon diversity index ( $\left.H^{\prime}\right)$ has 3.65 and the Pielou evenness $\left(J^{\prime}\right)$ of 0,775 . It was observed the presence of the diametric distribution pattern present in this inverted $J$ plant community.

Keywords: Survey phytosociological, inverted J, Cerrado. 
El objetivo fue determinar la estructura y fitosociológico diametral del stricto sensu un cerrado. Este estudio se llevó a cabo en el campus de la granja experimental Gurupí Reserva Legal UFT, y el sitio incluye el área de reserva legal de esta propiedad y se encuentra en las coordenadas geográficas $11^{\circ} 77^{\prime} 30.5^{\prime \prime} \mathrm{S}, 4^{\circ} 05^{\prime} 62.9^{\prime \prime} \mathrm{W}$. Sistemáticamente se han instalado cinco parcelas de $20 \times 50 \mathrm{~m}\left(1.000 \mathrm{~m}^{2}\right)$ cada una, espaciadas $10 \mathrm{~m}$ de distancia, para un total de $5000 \mathrm{~m}^{2}$, o área de muestra de 0,5 ha. Estas parcelas fueron muestreados cada individuos arbustivos arbórea con la circunferencia a la altura del pecho $(C A P) \geq 10 \mathrm{~cm}$. Se muestrearon 2,128 individuos, de los cuales 2.062 en vivo y de pie 66 muertos, distribuidos en 110 especies y 86 géneros y 46 familias. La altura media de la vegetación se estimó en 7,23 m, se encontró que el diámetro medio de 6,87 cm de estar, además de haber realizado un área basal total de 10,88 $\mathrm{m}^{2}$. 'Se encontró que era 3,65 y uniformidad de Pielou (J') El índice de diversidad de Shannon ( $\left.H^{\prime}\right)$ 0.775. Se observó la presencia de patrón de distribución de diámetros presente en esta comunidad vegetal, invertida $J$.

Descriptores: Estrutura vegetacional, J invertido, Cerrado.

\section{INTRODUÇÃO}

A vegetação brasileira é ainda pouco conhecida em parte devido à extensão territorial do País. O Cerrado é um bioma com divisões onde dentro do cerrado "sensu lato" se encontra o: Campo limpo, campo sujo, campo cerrado, cerrado sensu stricto e cerradão; é rico em biodiversidades onde está com cerca de $40 \%$ de sua cobertura original e apenas $2 \%$ de sua área protegida em unidades de conservação. $\mathrm{O}$ bioma Cerrado que, segundo (MYERS et al., 2000), possui uma biodiversidade riquíssima ainda parcialmente conhecida, sofre com o desmatamento e a intensificação da ocupação agropecuária e agrícola.

De acordo com (LEAK, 1964) e (MEYER, 1943, 1952, 1953), as formações florestais equilibradas apresentam a distribuição diamétrica dos indivíduos na forma exponencial negativa, ou seja, o histograma de frequências dos indivíduos se assemelha a um $\mathrm{j}$-invertido, sendo que a maior frequência de indivíduos se encontra nas classes de diâmetro menores.

Segundo (MEYER et al., 1961), a estrutura diamétrica reflete a história de uma comunidade vegetal e pode ser um indicativo de equilíbrio ou desequilíbrio (LEAK, 1964; HARPER, 1990) e de sua adaptação às modificações do ecossistema (FELFILI, 2001). As diferentes formações florestais apresentam distribuições diamétrica também diferentes, tanto na sua amplitude como na sua forma. Por isso, a distribuição de diâmetro é característica importante para a avaliação de estoque em crescimento (FERREIRA et al., 1998). De acordo com (PAULA et al., 2004), a distribuição diamétrica é uma das ferramentas utilizadas para a compreensão da sucessão e tem sido utilizada frequentemente em trabalhos de manejo florestal aplicado a povoamentos inequiâneos.

Outra forma de análise da estrutura arbórea em um fragmento é em relação à sua verticalização. A estratificação vertical ou hipsométrica é muito importante, seja para fins de estudos fitossociológicos, seja para fins de manejo florestal. Dependendo da região fitoecológica, do estágio de sucessão e do estado de conservação, a estrutura florestal pode ser agrupada em diferentes tipos de estratos de altura, por exemplo. Esse tipo de estratificação influencia a riqueza, a diversidade, o crescimento e a produção de biomassa, sendo um importante indicador de sustentabilidade ambiental de uma floresta (SOUZA et al., 2004). 
Objetivou-se analisar a fitossociologia de uma área de vegetação de cerrado sensu stricto e estimar a distribuição diamétrica dos indivíduos de cada espécie.

\section{MATERIAIS E MÉTODOS}

Foi realizado um levantamento florístico e fitossociológico de uma área de cerrado sensu stricto da Fazenda Experimental da Universidade Federal do Tocantins, campus de Gurupi-TO sob as coordenadas $11^{\circ} 46^{\prime} 21.5^{\prime \prime} \mathrm{S}$ e $49^{\circ} 03^{\prime} 21.9^{\prime \prime} \mathrm{W}$, com 299 m de altitude e o clima da região foi caracterizado segundo a classificação de Köppen do tipo mesotérmico com inverno seco (Cwa) e verão chuvoso (SEPLAN, 2012).

Foram instaladas cinco parcelas amostrais com dimensões de 20 x 50 m $\left(1000 \mathrm{~m}^{2}\right)$ cada, perfazendo um total de $5000 \mathrm{~m}^{2}$, ou seja, 0,5 ha de área amostral onde 10 subparcelas de 10 x $10 \mathrm{~m}$ $\left(100 \mathrm{~m}^{2}\right)$ foram demarcadas dentro de cada parcela, totalizando 50 subparcelas. A distribuição destas parcelas ocorreu de forma sistemática ao longo das áreas de estudo de cerrado sensu stricto, sendo distanciadas $10 \mathrm{~m}$ entre si. O tamanho das parcelas utilizada neste trabalho está padronizado segundo o projeto Biogeografia do Bioma Cerrado (FELFILI e SILVA JUNIOR, 1992; FELFILI et al., 1997).

Foram amostrados todos os indivíduos arbustivo-arbóreos com circunferência a $1,30 \mathrm{~m}$ do solo (CAP) maior ou igual a $10 \mathrm{~cm}$, visando contribuir para o conhecimento do domínio do Cerrado Tocantinense e com os dados foi feita a distribuição diamétrica por espécies provenientes desse levantamento.

A identificação taxonômica das espécies presentes na área de estudo foi realizada através de comparações com o material do herbário de Porto
Nacional, da Universidade Federal do Tocantins, literatura especializada e consulta a especialistas, quando necessário.

Foi utilizado o sistema Angiosperm Phylogeny Group III para classificação das famílias botânicas (APG III, 2009). Todos os nomes das espécies e seus respectivos autores e sinonímias foram confirmados e atualizados pelo site da Lista de Espécies da Flora do Brasil http://reflora.jbrj.gov.br/ (REFLORA DO BRASIL, 2015).

Os dados básicos obtidos das 50 unidades amostrais de 0,01 ha cada, foram analisados para fins de obtenção dos parâmetros fitossociológicos: densidade relativa (DR), Densidade absoluta (DA), dominância relativa (DOR), dominância absoluta (DoA), frequência relativa (FR), frequência absoluta (FA) e índice de valor de importância (IVI), foram calculados pelas formas tradicionais, além dos índices de diversidade de Shannon (H') e 23 equabilidade de Pielou (J'), a partir do uso do programa Fitopac versão 2.1.2 (SHEPPERD, 2010).

Os critérios de agrupamentos foram a presença simultânea dos indivíduos, por espécie, em sucessivas classes com diâmetro e o tamanho máximo, que os indivíduos das espécies alcançaram. A presença simultânea dos indivíduos em sucessivas classes diamétricas propiciou separar as espécies em grupos ecológicos, cuja distribuição diamétrica foi continua ou descontinua. O critério de máximo tamanho permite estabelecer a amplitude da distribuição diamétrica de cada grupo (SOUZA e JESUS, 1994).

A distribuição diamétrica foi feita mediante o cômputo dos indivíduos amostrados de cada espécie dentro da classe diamétrica a que pertencessem (HARPER, 1977). As classes de diâmetro foram estabelecidas com amplitude de $5 \mathrm{~cm}$, a partir do 
diâmetro mínimo de $3 \mathrm{~cm}$ e as classes de altura foram estabelecidas com amplitude de $3 \mathrm{~m}$ a partir da altura mínima de 1,3 m. Para obtenção dos gráficos de distribuição diamétrica foi utilizado o programa MSExcel (Microsoft. 2010).

\section{RESULTADOS E DISCUSSÃO}

Foram amostrados em 0,5ha um total de 2128 indivíduos, dos quais 2062 vivos e 66 mortos em pé, distribuídos em 110 espécies 86 gêneros e 46 famílias.

As árvores mortas em pé, totalizaram 3,1\% dos indivíduos amostrados, valor este que corrobora com (CAVASSAN, 1982) onde encontrou 5,8\% de árvores mortas, o que é normal em florestas nativas. Como apresentou elevada frequência, ocorrendo em $100 \%$ das parcelas, indica que não está havendo uma perturbação localizada, pois a morte das árvores pode estar relacionada com acidentes como: ventos, tempestades, queda de grandes ramos, doenças, perturbações antrópicas (fogo) e até mesmo morte natural dos indivíduos mais velhos (MARTINS, 1991; SILVA e SOARES, 2002).

O índice de diversidade de Shannon Weaver (H') encontrado foi de 3.65 valores semelhantes aos encontrados por, (FERREIRA et al., 2015) que foi de 3.70 . Segundo (PIELOU, 1975) e (MARTINS, 1991), os valores de diversidade de Shannon variam de 1,5 a 3,5 nats/ind., raramente passando de 4,5.

A equabilidade de Pielou (J') encontrada foi de 0,77 o que indica uma heterogeneidade florística relativamente alta do componente arbóreo 77\% daquela máxima possível (MAGURRAN, 1988; LOPES et al., 2002). Este resultado demonstra que a área estudada apresenta alta diversidade, com baixa dominância ecológica (GIÁCOMO et al., 2013). O diâmetro médio encontrado foi de $6,87 \mathrm{~cm}$ e uma área basal de $10,88 \mathrm{~m}^{2}$.

As famílias Anacardiaceae, Vochysiaceae, Burseraceae, Fabaceae, Myrtaceae, Sapindaceae, Rubiaceae e Combretaceae obtiveram $100 \%$ de frequência absoluta indicando uma ampla distribuição dessas famílias na área. Dados estes que corroboram com (NERI et al., 2007) onde encontraram as famílias Vochysiaceae, Fabaceae e Malpighiaceae com $100 \%$ de frequência em um levantamento realizado em um cerrado sensu stricto de Minas Gerais.

As famílias que apresentaram os maiores índices de valor de importância foram Anacardiaceae $(12,97 \%)$, Fabaceae (11,36\%), Vochysiaceae $(7,12 \%)$, Burseraceae (6,55\%), Myrtaceae $(5,85 \%)$, Rubiaceae $(4,84 \%)$, Sapindaceae $(4,56 \%)$ e Malvaceae $(4,33 \%)$ somando juntas $57,58 \%$ da soma total do IVI. Os resultados encontrados no presente estudo corroboram com os encontrados por (FINGER et al., 2015) onde os mesmos obtiveram as famílias Fabaceae, Myrtaceae e Vochysiaceae como as mais representativas.

$\mathrm{O}$ destaque de Fabaceae em riqueza de espécies já era esperado, uma vez que essa família tem se destacado na maioria dos levantamentos realizados em cerrados, cerradões e Florestas Semidecíduas (CAMPOS et al., 2006; SOUZA et al., 2012). Essa vantagem competitiva das Fabaceae pode ser atribuída à capacidade de fixação biológica de nitrogênio de muitas espécies desta família, facilitando a regeneração em solos pobres e degradados, o que demonstra uma alta aptidão de uso de espécies desta família para recuperação de áreas degradadas (CAMPELLO, 1998).

Além da Fabaceae, as famílias Myrtaceae e Vochysiaceae apresentaram valores altos de riqueza, 
dados estes que corroboram com (NERI et al., 2007). Já (LEITÃO FILHO, 1992), em um estudo da flora arbórea de Cerrado no Estado de São Paulo destacaram a família Myrtaceae como detentora do maior número de espécies.

Foram analisados para fins de obtenção da matriz que relaciona o número de árvores, por hectare da i-ésima espécie na j-ésima classe de diâmetro. Diante do contexto a matriz constituiu-se da distribuição do número de árvores por hectare, ou seja, 2128 arvores representadas em 111 espécies distribuídas em 9 classes de diâmetro, com $5 \mathrm{~cm}$ de amplitude desde $3,18 \mathrm{~cm}$ até a classe de $47,9 \mathrm{~cm}$ sendo que a classe de 38 a 42,9 cm não foi encontrado nenhum indivíduo (Figura 1).

A distribuição dos indivíduos nas classes diamétricas apresentaram um padrão típico de Jinvertido ou exponencial negativa, ou seja, alta concentração de indivíduos nas classes de menor diâmetro e redução acentuada no sentido das classes maiores, supondo que as populações que compõe uma comunidade são estáveis e auto-regenerativas e que existe um balanço entre a mortalidade e o recrutamento dos indivíduos (Figura 1).

Figura 1. Distribuição diamétrica dos indivíduos de uma área de cerrado sensu stricto Gurupi-TO.

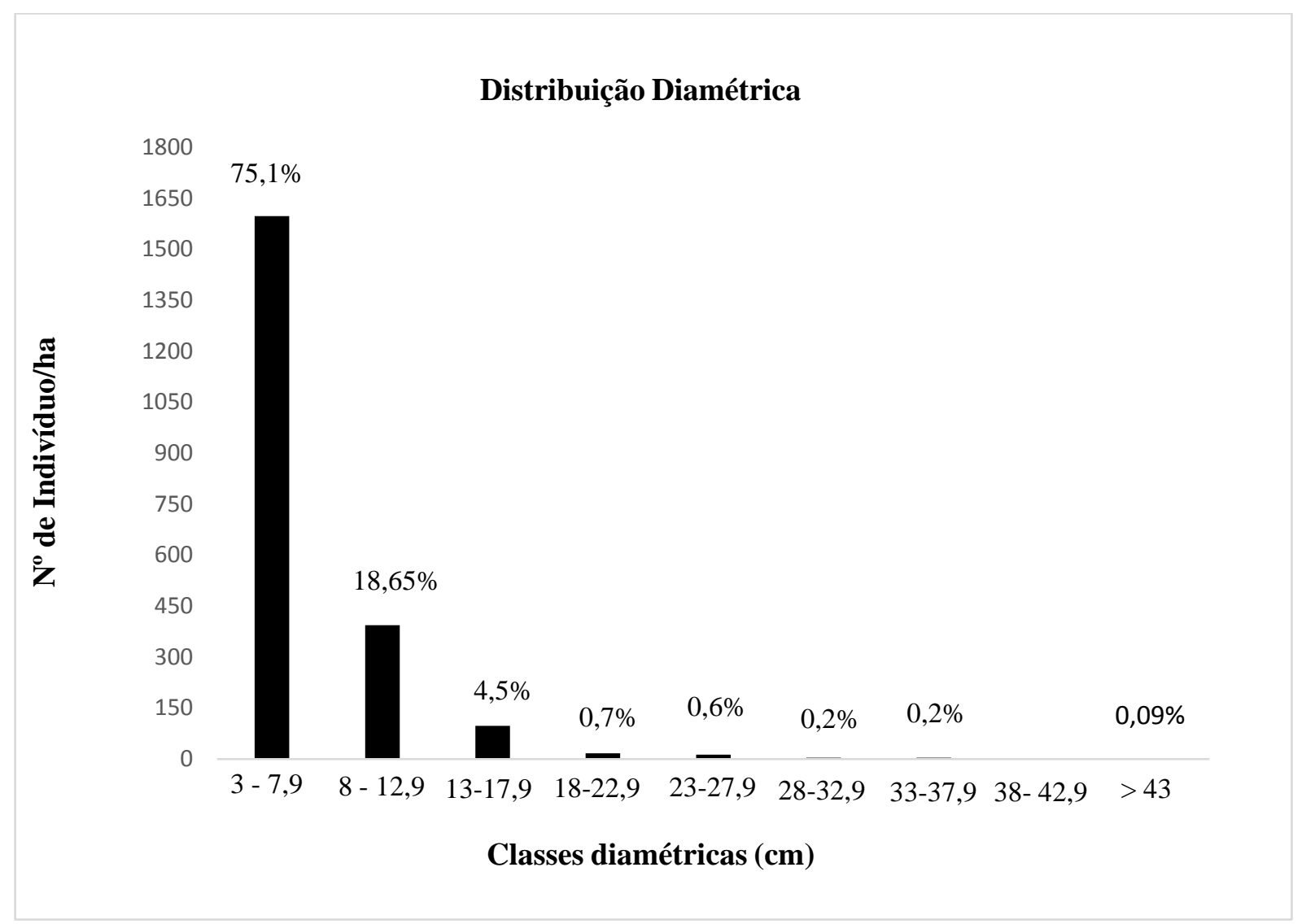


Este tipo de distribuição indica que novos indivíduos estão estabelecendo-se na área em proporção pequena, o que pode ser relacionado a necessidade de condições específicas de regeneração como por exemplo a abertura de clareiras e ao consequentemente aumento de luminosidade. A maior concentração de indivíduos nas primeiras classes de diâmetro pode caracterizar uma comunidade estoque, o que é padrão em florestas tropicais estáveis com idade e composição de espécies variadas.

Espécies com distribuição diamétrica errática, ou descontínua, englobam todo o gradiente ecofisiológico, ou seja, as pioneiras, as oportunistas, as secundárias iniciais, as secundarias tardias e as clímax, (SOUZA et al., 2012)

A classe de diâmetro de $3 \mathrm{~cm}$ a $8 \mathrm{~cm}$ centímetros compreendeu o maior número de indivíduos nesse estrato, sendo $75,1 \%$ dos indivíduos. De acordo com os dados (figura 1), observa-se que ocorre uma elevada concentração de indivíduos na menor classe diamétrica, entretanto cabe ressaltar que os mesmos pertencem aos grupos ecológicos das espécies secundarias iniciais e tardias, o que indica uma elevada regeneração devido à grande entrada de indivíduos e, consequentemente um promissor avanço para estágios sucessionais maduros.

Verifica-se que 1597 indivíduos entraram na primeira classe de diâmetro 3 a 7,9 $\mathrm{cm}$ perfazendo um total de $75,1 \%$ dos indivíduos amostrados na área de estudo, 394 indivíduos estão presentes na classe de diâmetro entre 8 a 12,9 $\mathrm{cm}$ perfazendo um total de 18,5\%, 97 indivíduos estão presentes nas classe de diâmetro de 13 a $17,9 \mathrm{~cm}$ ou seja 4,5\% do total dos indivíduos amostrados os demais indivíduos apresentaram porcentagem menores que $1 \%$, (ASSUNÇÃO e FELFILI, 2004) no APA do lago
Paranoá encontrou 95\% dos indivíduos com diâmetro até $25 \mathrm{~cm}$,a área de cerrado estudada apresentou 99,4\% dos indivíduos com diâmetros até 25 padrão confirmado no cerrado sensu stricto (FELFILI, 2004).

Foi observado que $81,55 \%$ dos indivíduos estão agrupados nas três primeiras classes de altura foi observado que a classe 2 entre 6,1 a 9 metros apresentou maior número de indivíduos, representando $42,8 \%$ do total, fato esse que pode ser comprovado que a maioria dos indivíduos está representada na classe de altura de 3 a 9 metros (figura 2). Com o estudo da estrutura vertical, é possível analisar o estágio de desenvolvimento da floresta, com base na distribuição dos indivíduos nos diferentes estratos, juntamente com os dados obtidos na distribuição diamétrica (MARANGON et al., 2008).

Figura 2. Distribuição diamétrica das classes de altura dos indivíduos de uma área de cerrado sensu stricto Gurupi - TO

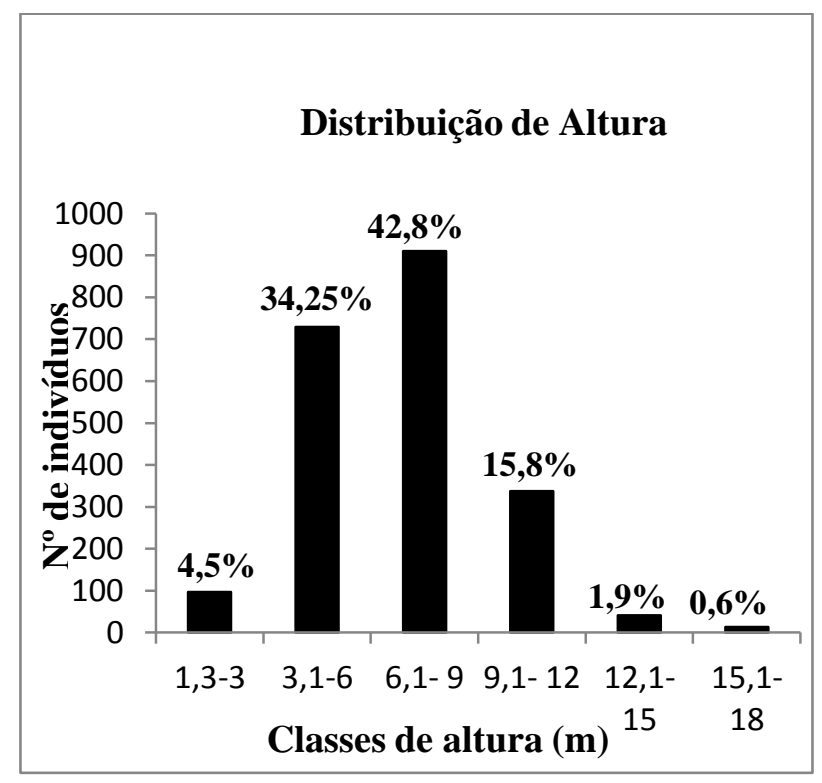

Evidenciou-se que a área de estudo cerrado sensu stricto está passando por um processo de transição entre fisionomias do bioma cerrado, ou seja, 
a área de estudo está em processo de sucessão de fisionomia aberta cerrado sensu stricto para formação florestal cerradão, uma vez que os indivíduos amostrados apresentaram distribuição diamétrica de suas alturas concentradas nas classes intermediárias, ou seja, altura média de 7,23 m confirmando que área estudada está muito próxima do critério de inclusão usualmente adotado para classificar as formações florestais (cerradão) presente no domínio Cerrado que é altura do dossel de 8 a 13 m (RATTER et al., 2006), fato esse comprovado num estudo realizado em Rio Verde - GO onde a distribuição das alturas se concentraram nas classes intermediárias (SOARES et al., 2012), além de corroborarem com dados encontrados no APA do lago Paranoá em Brasília por (ASSUNÇÃO e FELFILI, 2004) e (FERREIRA, 2015) em Gurupi-TO.

\section{CONCLUSÃO}

A observação dos parâmetros fitossociológicos permite a recomendação de algumas Famílias com potencial para a recuperação de fragmentos de cerrado sensu stricto da região sul do Estado do Tocantins.

A análise da estrutura diamétrica da fisionomia cerrado sensu stricto analisada pode contribuir para o manejo florestal.

A distribuição dos indivíduos arbustivoarbóreos dos quatro estratos nas classes diamétricas apresentou padrão típico de J-invertido.

A elevada concentração de indivíduos classificadas na classe sucessional como secundárias iniciais e tardias indica que a área está em elevada taxa de regeneração, devido à grande quantidade de indivíduos, consequentemente é um promissor avanço para estágios sucessionais maduros.

\section{REFERENCIAS BIBLIOGRAFICAS}

ASSUÇÃO, S. LFELFILI, J. M.;. Fitossociologia de um fragmento de cerrado sensu stricto na APA do Paranoá, DF, Brasil: Acta Boânica Brasilica, Brasilia, v.18, n.4 p.903-909, 2004.

ANGIOSPERM PHYLOGENY GROUP - APG. An update of the Angiosperm Phylogeny Group classification for the orders and families of flowering plants: APG III. Bot. J. Linn. Soc., v.161, p.105-121. 2009.

CAMPELlO, E. F. C. Sucessão vegetal na recuperação de áreas degradadas. In: DIAS, L. E; MELLO, L. W. V. Recuperação de áreas degradadas. Viçosa: SOBRADE, 1998. p. 183-196.

CAMPOS, É. P; DUARTE, T. G; NERI, A. V.; SILVA, A. F.; MEIRA NETO, J. A. A.; VALENTE, G. E. Composição florística de um trecho de cerradão e cerrado sensu stricto e sua relação com o solo na Floresta Nacional de Paraopeba, MG, Brasil. Revista Árvore, Viçosa, v. 30, n. 6, p. 471-479, 2006.

CAVASSAN, O. Levantamento fitossociológico da vegetação arbórea da mata da Reserva Estadual de Bauru utilizando o método de quadrantes. 1982. 102 p. Dissertação (Mestrado em Ciências Biológicas) Instituto de Biociências de Rio Claro, Universidade Estadual Paulista Julio de Mesquita Filho, Rio Claro.

FELFILI, J. M. Composição florística e fitossociologia de um cerrado Sensu Stricto em Agua Boa-MT. Acta Botânica Brasilica, Brasília , v.16, n. 5 p.103-112,2002.

FELFILI, J. M.; SILVA JÚNIOR, M.C. Biogeografia do Bioma Cerrado: estudo fitofisionômico na Chapada do Espigão Mestre do São Francisco. Brasília: Universidade de Brasília, 2001. 152p.

FELFILI, J. M.; SILVA JÚNIOR, M. C.; REZENDE, A. V.; NOGUEIRA, P. E.; WALTER, B. M. T.; SILVA, M.; ENCINAS, J. I. Comparação florística e fitossociológica do Cerrado nas Chapadas Pratinha e dos Veadeiros. Brasília: Ed. UNB, 1997.

FELFILI, J. M.; SILVA JÚNIOR, M. C. Floristic composition, phytosociology and comparison of cerrado and gallery forests at Fazenda Água Limpa, Federal District, Brazil. Pp. 393-415. In: P. A. Furley; J. A. Proctor \& J. A. Ratter. Nature and 
dynamics of forest-savanna boudaries, London, Chapman \& Hall, 1992.

FERREIRA, R. L. C.; SOUZA, A. L.; JESUS, R. M. Dinâmica da estrutura de uma floresta secundária de transição. II - Distribuição diamétrica. Revista Árvore, Viçosa-MG, v. 22, n. 3, p. 331-344, 1998.

FINGER, Z.; FINGER, F. A. Fitossociologia em comunidades arbóreas remanescentes de cerrado sensu stricto no brasil central: FLORESTA, Curitiba, PR, v. 45, n. 4, p. $769-780,2015$.

LEAK W. Na expression of diameter distribution for unbalanced, unvenaged stands and forests. Science 1964; v10: 39-50

LEITÃO FILHO, H.F. A flora arbórea da Serra do Japi. In: MORELLATO, L.P.C. História Natural da Serra do Japi: ecologia e preservação de uma área florestal no Sudeste do Brasil. Campinas: Editora da UNICAMP, 1992. p.40-62.

MARAGON, L. C.; FELICIANO, A. L. P.; BRANDÃO, C. F. L. S.; ALVES JUNIOR, F. T. Relações florísticas, estrutura diamétrica e hipsométrica de um fragmento de floresta estacional semidecidual em viçosa(MG). Revista Floresta, v.38, n.4, 2008.

MARTINS, F. R. Estrutura de uma floresta mesófila. São Paulo: Ed da UNICAMP, 1991. 246 p.

MEYER AH, RICKNAGEL AB, STEVENSON DD, BARTOO RA. Forest management. New York: The Ronald Press Company; 1961.

MEYER HA. Forest mensuration. State College, Pa: Renns Valley Publishers; 1953.

MEYER HA. Management without rotation. Journal of Forestry 1943; 41: 126-132.

MEYER, H. A. Structure, growth, and drain in balanced uneven-aged forests. Journal of Forestry, Bethesda, n. 52, v. 2, p. 85-92, 1952.

MUELLER-DOMBOIS, D. Y.; ELLENBERG, M. Aims and methods in vegetation ecology. New York: John Wiley \& Sons, 1974. 547 p.
MYERS N, MITTERMELER RA, MITTERMELER CG, FONSECA GAB, KENTS J. Bioversity hotspots for conservation priorities. Nature 2000; 403: p. 853858.

NERI, V. A; MEIRA NETO, J. A. A; SILVA, A. F; MARTINS, S. V; SAPORETTI JUNIOR, A. W. Composição florística de uma área de cerrado sensu stricto no município de Senador Modestino Gonçalves, Vale do Jequitinhonha (MG) e análise de similaridade florística de algumas áreas de cerrado em Minas Gerais. Revista. Árvore, Viçosa, v.31, n.6, p.1109-1119, 2007.

PAULA A, SILVA AF, MARCO JÚNIOR P, SANTOS FAM, SOUZA AL. Sucessão ecológica da vegetação arbórea em uma floresta estacional semidecidual, Viçosa, MG, Brasil. Acta Botanica Brasilica ; v.18 , n.3 , p.407-423, 2004.

PIELOU, E. C. Ecological diversity. New York: John Wiley, 1975. 165 p.

RATTER, J. A.; BRIDGEWATER, S.; RIBEIRO, F. Biodiversity patterns of the woody vegetation of the Brazilian Cerrado. In: PENNINGTON; R.T.; LEWIS, G.P.; RATTER, J.A. (Eds.) Neotropical savannas and seasonally dry forests: plant diversity, biogeography and conservation. London, Taylor e Francis, 2006. p.31-66

SEPLAN. Atlas do Tocantins: subsídios ao planejamento da gestão territorial. 6. ed. Palmas: Secretaria do Planejamento e da Modernização da Gestão Pública, 80 p. 2012.

SHEPERD, G. J. FITOPAC 2: manual do usuário. Campinas: UNICAMP, 2010. 91p.

SILVA JÚNIOR, M.C. et al. Análise da flora arbórea de Matas de Galeria no Distrito Federal: 21 levantamentos. In: RIBEIRO, J.F.; FONSECA, C.E.L.; SOUSA-SILVA, J.C. Cerrado: caracterização e recuperação de Matas de Galeria. Planaltina, DF: EMBRAPA Cerrados, p. 142-191, 2001.

SILVA, L. A; SOARES, J. J. Levantamento fitossociológico em um fragmento de floresta estacional semidecídua no município de São Carlos, SP. Acta botânica brasílica, Feira de Santana, v.16, n. 2, p. 205-216, 2002. 
SOARES, M. P.; SANTOS, T. M.; DOURADO, D. M.; SILVA, P. O.; AS, J. L. Analise fitossociologica do componente arbóreo de um remanescente de cerradão em rio verde -Go. Revista GI.Sci technology, v.05, n. 03, p. 87-97, 2012.

SOUZA, P. B. Composição florística do estrato arbóreo e estrutura de uma área de cerradão na floresta nacional de Paraopeba, Minas Gerais. 61 p. Dissertação (mestrado em Botânica) Departamento de Botânica - Universidade Federal de Viçosa, viçosa, 2004.

SOUZA, V, C. LORENZI, H. Botânica sistemática: Guia ilustrado para a identificação de famílias de angiospermas, baseado em APG II. Nona Odessa SP, Editora: Instituto Plantarum, 2005.

SOUZA. P, B.; SOUZA A, L.; MEIRA NETO, J , A, A.Estrutura diamétrica dos estratos e grupos ecológicos de uma área de Floresta Estacional Semidecidual,em Dionísio, MG. Revista Arvore. Viçosa-MG, vol.36 no.1, 2012 